\title{
Positive solutions for fractional differential equations with three-point multi-term fractional integral boundary conditions
}

\author{
Jessada Tariboon ${ }^{1 *}$, Sotiris K Ntouyas ${ }^{2}$ and Weerawat Sudsutad ${ }^{1}$
}

\author{
"Correspondence: \\ jessadat@kmutnb.ac.th \\ 'Department of Mathematics, \\ Faculty of Applied Science, King \\ Mongkut's University of Technology \\ North Bangkok, Bangkok, Thailand \\ Full list of author information is \\ available at the end of the article
}

\begin{abstract}
We are concerned with the existence of at least one, two or three positive solutions for the boundary value problem with three-point multi-term fractional integral boundary conditions:

$$
\left\{\begin{array}{l}
D^{q} u(t)+f(t, u(t))=0, \quad 1<q \leq 2,0<t<1, \\
u(0)=0, \quad u(1)=\sum_{i=1}^{m} \alpha_{i}\left(p_{i} u\right)(\eta), \quad 0<\eta<1,
\end{array}\right.
$$

where $D^{q}$ is the standard Riemann-Liouville fractional derivative. Our analysis relies on the Krasnoselskii fixed point theorem and the Leggett-Williams fixed point theorem. Some examples are also given to illustrate the main results.

MSC: 26A33; 34A08; 34B18
\end{abstract}

Keywords: fractional differential equations; nonlocal boundary conditions; positive solutions; fixed point theorem

\section{Introduction}

In recent years, the interest in the study of fractional differential equations has been growing rapidly. Fractional differential equations have arisen in mathematical models of systems and processes in various fields such as aerodynamics, acoustics, mechanics, electromagnetism, signal processing, control theory, robotics, population dynamics, finance, etc.

We refer a reader interested in the systematic development of the topic to the books [1-7]. A variety of results on initial and boundary value problems of fractional differential equations and inclusions can easily be found in the literature on the topic. For some recent results, we can refer to [8-19] and references cited therein.

In this paper, we concentrate on the study of positive solutions to the boundary value problems of fractional differential equations. More precisely, we consider the nonlinear fractional differential equation

$$
D^{q} u(t)+f(t, u(t))=0, \quad 1<q \leq 2,0<t<1,
$$

subject to three-point multi-term fractional integral boundary conditions

$$
u(0)=0, \quad u(1)=\sum_{i=1}^{m} \alpha_{i}\left(I^{p_{i}} u\right)(\eta), \quad 0<\eta<1,
$$

\section{Springer}

(02014 Tariboon et al.: licensee Springer. This is an Open Access article distributed under the terms of the Creative Commons Attribution License (http://creativecommons.org/licenses/by/2.0), which permits unrestricted use, distribution, and reproduction in any medium, provided the original work is properly cited. 
where $D^{q}$ is the standard Riemann-Liouville fractional derivative of order $q, I^{p_{i}}$ is the Riemann-Liouville fractional integral of order $p_{i}>0, i=1,2, \ldots, m, f:[0,1] \times[0, \infty) \rightarrow$ $[0, \infty)$ and $\alpha_{i} \geq 0, i=1,2, \ldots, m$, are real constants such that $\sum_{i=1}^{m} \frac{\alpha_{i} \eta^{p_{i}+q-1} \Gamma(q)}{\Gamma\left(p_{i}+q\right)}<1$.

We mention that integral boundary conditions are encountered in various applications such as population dynamics, blood flow models, chemical engineering, cellular systems, heat transmission, plasma physics, thermoelasticity, etc. Nonlocal conditions come up when values of the function on the boundary is connected to values inside the domain.

One of the most frequently used tools for proving the existence of positive solutions to the integral equations and boundary value problems is the Krasnoselskii theorem on cone expansion and compression and its norm-type version due to Guo and Lakshmikantham [20]. The main idea is to construct a cone in a Banach space and a completely continuous operator defined on this cone based on the corresponding Green's function and then find fixed points of the operator. See $[9,18]$ and references therein for recent development.

The rest of this paper is organized as follows. In Section 2 we present some necessary basic knowledge and definitions for fractional calculus theory and give the corresponding Green's function of boundary value problem (1.1)-(1.2). Moreover, some properties of the Green's function are also proved. In Section 3 we use the properties of the corresponding Green's function and the Guo-Krasnoselskii fixed point theorem to show the existence of at least one or two positive solutions of (1.1)-(1.2) under the condition that the nonlinear $f$ is either sublinear or superlinear. In Section 4 we prove the existence of at least three positive solutions via the Leggett-Williams fixed point theorem. Finally, illustrative examples are presented in Section 5 .

\section{Preliminaries}

In this section, we introduce some notations and definitions of fractional calculus [3, 4] and present preliminary results needed in our proofs later.

Definition 2.1 The Riemann-Liouville fractional integral of order $\alpha>0$ of a function $g$ : $(0, \infty) \rightarrow \mathbb{R}$ is defined by

$$
I^{\alpha} g(t)=\int_{0}^{t} \frac{(t-s)^{\alpha-1}}{\Gamma(\alpha)} g(s) d s
$$

provided the right-hand side is point-wise defined on $(0, \infty)$, where $\Gamma$ is the gamma function.

Definition 2.2 The Riemann-Liouville fractional derivative of order $\alpha>0$ of a continuous function $g:(0, \infty) \rightarrow \mathbb{R}$ is defined by

$$
D^{\alpha} g(t)=\frac{1}{\Gamma(n-\alpha)}\left(\frac{d}{d t}\right)^{n} \int_{0}^{t} \frac{g(s)}{(t-s)^{\alpha-n+1}} d s, \quad n-1<\alpha<n,
$$

where $n=[\alpha]+1,[\alpha]$ denotes the integer part of a real number $\alpha$, provided the right-hand side is point-wise defined on $(0, \infty)$.

From the definition of the Riemann-Liouville fractional derivative, we can obtain the following lemmas. 
Lemma 2.1 (see [4]) Let $\alpha>0$ and $y \in C(0,1) \cap L(0,1)$. Then the fractional differential equation $D^{\alpha} y(t)=0$ has a unique solution

$$
y(t)=c_{1} t^{\alpha-1}+c_{2} t^{\alpha-2}+\cdots+c_{n} t^{\alpha-n},
$$

where $c_{i} \in \mathbb{R}, i=1,2, \ldots, n$, and $n-1<\alpha<n$.

Lemma 2.2 (see [4]) Let $\alpha>0$. Then for $y \in C(0,1) \cap L(0,1)$ it holds

$$
I^{\alpha} D^{\alpha} y(t)=y(t)+c_{1} t^{\alpha-1}+c_{2} t^{\alpha-2}+\cdots+c_{n} t^{\alpha-n},
$$

where $c_{i} \in \mathbb{R}, i=1,2, \ldots, n$, and $n-1<\alpha<n$.

The following property (Dirichlet's formula) of the fractional calculus is well known [1]:

$$
I^{\gamma} I^{\beta} u(t)=I^{\gamma+\beta} u(t), \quad t \in[0,1], u \in L(0,1), \gamma+\beta \geq 1,
$$

which has the form

$$
\int_{0}^{t}(t-s)^{\gamma-1}\left(\int_{0}^{s}(s-\tau)^{\beta-1} u(\tau) d \tau\right) d s=\frac{\Gamma(\gamma) \Gamma(\beta)}{\Gamma(\gamma+\beta)} \int_{0}^{t}(t-s)^{\gamma+\beta-1} u(s) d s .
$$

For convenience we put

$$
\Omega:=1-\sum_{i=1}^{m} \frac{\alpha_{i} \eta^{p_{i}+q-1} \Gamma(q)}{\Gamma\left(p_{i}+q\right)}
$$

Lemma 2.3 Let $\sum_{i=1}^{m} \frac{\alpha_{i} \eta^{p_{i}+q-1} \Gamma(q)}{\Gamma\left(p_{i}+q\right)}<1, \alpha_{i} \geq 0, p_{i}>0, i=1,2, \ldots, m$, and $h \in C([0,1], \mathbb{R})$. The unique solution $u \in A C([0,1], \mathbb{R})$ of the boundary value problem

$$
\begin{aligned}
& D^{q} u(t)+h(t)=0, \quad t \in(0,1), q \in(1,2], \\
& u(0)=0, \quad u(1)=\sum_{i=1}^{m} \alpha_{i}\left(I^{p_{i}} u\right)(\eta), \quad 0<\eta<1,
\end{aligned}
$$

is the integral equation

$$
u(t)=\int_{0}^{1} G(t, s) h(s) d s
$$

where $G(t, s)$ is the Green's function given by

$$
G(t, s)=g(t, s)+\sum_{i=1}^{m} \frac{\alpha_{i} t^{q-1}}{\Omega \Gamma\left(p_{i}+q\right)} g_{i}(\eta, s)
$$

where

$$
g(t, s)= \begin{cases}\frac{t^{q-1}(1-s)^{q-1}-(t-s)^{q-1}}{\Gamma(q)} ; & 0 \leq s \leq t \leq 1, \\ \frac{t^{q-1}(1-s)^{q-1}}{\Gamma(q)} ; & 0 \leq t \leq s \leq 1\end{cases}
$$


and

$$
g_{i}(\eta, s)= \begin{cases}\eta^{p_{i}+q-1}(1-s)^{q-1}-(\eta-s)^{p_{i}+q-1} ; & 0 \leq s \leq \eta<1 \\ \eta^{p_{i}+q-1}(1-s)^{q-1} ; & 0<\eta \leq s \leq 1\end{cases}
$$

Proof Using Lemmas 2.1-2.2, problem (2.2)-(2.3) can be expressed as an equivalent integral equation

$$
u(t)=c_{1} t^{q-1}+c_{2} t^{q-2}-\int_{0}^{t} \frac{(t-s)^{q-1}}{\Gamma(q)} h(s) d s
$$

for $c_{1}, c_{2} \in \mathbb{R}$. The first condition of (2.3) implies that $c_{2}=0$. Taking the Riemann-Liouville fractional integral of order $p_{i}>0$ for (2.8) and using Dirichlet's formula, we get that

$$
\begin{aligned}
\left(I^{p_{i}} u\right)(t) & =\int_{0}^{t} \frac{(t-s)^{p_{i}-1}}{\Gamma\left(p_{i}\right)}\left(c_{1} s^{q-1}-\int_{0}^{s} \frac{(s-r)^{q-1}}{\Gamma(q)} h(r) d r\right) d s \\
& =c_{1} \int_{0}^{t} \frac{(t-s)^{p_{i}-1} s^{q-1}}{\Gamma\left(p_{i}\right)} d s-\int_{0}^{t} \frac{(t-s)^{p_{i}-1}}{\Gamma\left(p_{i}\right)} \int_{0}^{s} \frac{(s-r)^{q-1}}{\Gamma(q)} h(r) d s d r \\
& =c_{1} \frac{t^{p_{i}+q-1} \Gamma(q)}{\Gamma\left(p_{i}+q\right)}-\frac{1}{\Gamma\left(p_{i}+q\right)} \int_{0}^{t}(t-s)^{p_{i}+q-1} h(s) d s .
\end{aligned}
$$

The second condition of (2.3) yields

$$
\begin{aligned}
c_{1}-\int_{0}^{1} \frac{(1-s)^{q-1}}{\Gamma(q)} h(s) d s= & c_{1} \sum_{i=1}^{m} \frac{\alpha_{i} \eta^{p_{i}+q-1} \Gamma(q)}{\Gamma\left(p_{i}+q\right)} \\
& -\sum_{i=1}^{m} \frac{\alpha_{i}}{\Gamma\left(p_{i}+q\right)} \int_{0}^{\eta}(\eta-s)^{p_{i}+q-1} h(s) d s .
\end{aligned}
$$

Then we have that

$$
c_{1}=\frac{1}{\Omega}\left[\int_{0}^{1} \frac{(1-s)^{q-1}}{\Gamma(q)} h(s) d s-\sum_{i=1}^{m} \frac{\alpha_{i}}{\Gamma\left(p_{i}+q\right)} \int_{0}^{\eta}(\eta-s)^{p_{i}+q-1} h(s) d s\right] .
$$

Therefore, the unique solution of boundary value problem (2.2)-(2.3) is written as

$$
\begin{aligned}
u(t)= & -\int_{0}^{t} \frac{(t-s)^{q-1}}{\Gamma(q)} h(s) d s+\frac{1}{\Omega \Gamma(q)} \int_{0}^{1}(1-s)^{q-1} t^{q-1} h(s) d s \\
& -\frac{1}{\Omega} \sum_{i=1}^{m} \frac{\alpha_{i}}{\Gamma\left(p_{i}+q\right)} \int_{0}^{\eta}(\eta-s)^{p_{i}+q-1} t^{q-1} h(s) d s .
\end{aligned}
$$

Hence, by taking into account (2.1), we have

$$
\begin{aligned}
u(t)= & -\int_{0}^{t} \frac{(t-s)^{q-1}}{\Gamma(q)} h(s) d s+\frac{1}{\Omega \Gamma(q)} \int_{0}^{1}(1-s)^{q-1} t^{q-1} h(s) d s \\
& -\frac{1}{\Omega} \sum_{i=1}^{m} \frac{\alpha_{i}}{\Gamma\left(p_{i}+q\right)} \int_{0}^{\eta}(\eta-s)^{p_{i}+q-1} t^{q-1} h(s) d s
\end{aligned}
$$




$$
\begin{aligned}
& +\int_{0}^{1} \frac{(1-s)^{q-1} t^{q-1}}{\Gamma(q)} h(s) d s-\int_{0}^{1} \frac{(1-s)^{q-1} t^{q-1}}{\Gamma(q)} h(s) d s \\
= & \int_{0}^{1} \frac{(1-s)^{q-1} t^{q-1}}{\Gamma(q)} h(s) d s-\int_{0}^{t} \frac{(t-s)^{q-1}}{\Gamma(q)} h(s) d s \\
& +\sum_{i=1}^{m} \frac{\alpha_{i} t^{q-1}}{\Omega \Gamma\left(p_{i}+q\right)}\left(\int_{0}^{1} \eta^{p_{i}+q-1}(1-s)^{q-1} h(s) d s-\int_{0}^{\eta}(\eta-s)^{p_{i}+q-1} h(s) d s\right) \\
= & \int_{0}^{1} g(t, s) h(s) d s+\int_{0}^{1} \sum_{i=1}^{m} \frac{\alpha_{i} t^{q-1}}{\Omega \Gamma\left(p_{i}+q\right)} g_{i}(\eta, s) h(s) d s \\
= & \int_{0}^{1} G(t, s) h(s) d s .
\end{aligned}
$$

The proof is completed.

Lemma 2.4 The Green's function $G(t, s)$ in (2.5) satisfies the following conditions:

$\left(\mathrm{P}_{1}\right) G(t, s)$ is continuous on $[0,1] \times[0,1]$;

$\left(\mathrm{P}_{2}\right) G(t, s) \geq 0$ for all $0 \leq s, t \leq 1$;

$\left(\mathrm{P}_{3}\right) G(t, s) \leq \max _{0 \leq t \leq 1} G(t, s) \leq g(s, s)+\sum_{i=1}^{m} \frac{\alpha_{i}}{\Omega \Gamma\left(p_{i}+q\right)} g_{i}(\eta, s)$ for all $s, t \in[0,1]$;

$\left(\mathrm{P}_{4}\right) \int_{0}^{1} \max _{0 \leq t \leq 1} G(t, s) d s \leq \frac{\Gamma(q)}{\Gamma(2 q)}+\sum_{i=1}^{m} \frac{\alpha_{i} p_{i}+q-1}{\Omega \Gamma\left(p_{i}+q\right)}\left(\frac{p_{i}+q(1-\eta)}{q\left(p_{i}+q\right)}\right)$;

$\left(\mathrm{P}_{5}\right) \min _{\eta \leq t \leq 1} G(t, s) \geq \sum_{i=1}^{m} \frac{\alpha_{i} \eta^{q-1}}{\Omega \Gamma\left(p_{i}+q\right)} g_{i}(\eta, s)$ for $s \in[0,1]$.

Proof It is easy to check that $\left(\mathrm{P}_{1}\right)$ holds. To prove $\left(\mathrm{P}_{2}\right)$, we will show that $g(t, s) \geq 0$ and $g_{i}(\eta, s) \geq 0, i=1,2, \ldots, m$, for all $0 \leq s, t \leq 1$.

Let $k_{1}(t, s)=t^{q-1}(1-s)^{q-1}-(t-s)^{q-1}$ for $0 \leq s \leq t \leq 1$, then we have

$$
k_{1}(t, s)=(t-s t)^{q-1}-(t-s)^{q-1} \geq(t-s)^{q-1}-(t-s)^{q-1}=0 .
$$

Let $k_{2}(t, s)=t^{q-1}(1-s)^{q-1}$ for $0 \leq t \leq s \leq 1$, then we get $k_{2}(t, s) \geq 0$. Therefore, $g(t, s) \geq 0$ for all $0 \leq s, t \leq 1$. Now, let $k_{3}^{i}(\eta, s)=\eta^{p_{i}+q-1}(1-s)^{q-1}-(\eta-s)^{p_{i}+q-1}$ for $0 \leq s \leq \eta<1$, then we get

$$
\begin{aligned}
k_{3}^{i}(\eta, s) & =\eta^{p_{i}+q-1}(1-s)^{q-1}-\eta^{p_{i}+q-1}\left(1-\frac{s}{\eta}\right)^{p_{i}+q-1} \\
& >\eta^{p_{i}+q-1}(1-s)^{q-1}-\eta^{p_{i}+q-1}(1-s)^{p_{i}+q-1} \\
& =\eta^{p_{i}+q-1}\left[(1-s)^{q-1}-(1-s)^{p_{i}+q-1}\right] \\
& >0 .
\end{aligned}
$$

Let $k_{4}^{i}(\eta, s)=\eta^{p_{i}+q-1}(1-s)^{q-1}$ for $0<\eta \leq s \leq 1$, then we have $k_{4}^{i}(\eta, s) \geq 0$. Therefore, $k_{3}^{i}(\eta, s), k_{4}^{i}(\eta, s) \geq 0, i=1,2, \ldots, m$, which implies that $g_{i}(\eta, s) \geq 0, i=1,2, \ldots, m$, for all $0 \leq s \leq 1$.

To prove $\left(\mathrm{P}_{3}\right)$, we will show that $g(t, s) \leq g(s, s)$ for $s, t \in[0,1]$. For $0 \leq s \leq t \leq 1$, by the definition of $k_{1}(t, s)$, we have

$$
\begin{aligned}
\frac{\partial}{\partial t} k_{1}(t, s) & =(q-1) t^{q-2}(1-s)^{q-1}-(q-1)(t-s)^{q-2} \\
& =(q-1)(t-t s)^{q-2}(1-s)-(q-1)(t-s)^{q-2}
\end{aligned}
$$




$$
\begin{aligned}
& \leq(q-1)(t-s)^{q-2}(1-s)-(q-1)(t-s)^{q-2} \\
& =-s(q-1)(t-s)^{q-2} \leq 0 .
\end{aligned}
$$

Hence, $k_{1}(t, s)$ is decreasing with respect to $t$. Then we have $g(t, s) \leq g(s, s)$ for $0 \leq s \leq t \leq 1$. For $0 \leq t \leq s \leq 1$, by the definition of $k_{2}(t, s)$, we have that $k_{2}(t, s)$ is increasing with respect to $t$. Thus $g(t, s) \leq g(s, s)$ for $0 \leq t \leq s \leq 1$. Therefore, $g(t, s) \leq g(s, s)$ for $0 \leq s, t \leq 1$.

From the above analysis, we have for $0 \leq s \leq 1$ that

$$
\begin{aligned}
G(t, s) & \leq \max _{0 \leq t \leq 1} G(t, s)=\max _{0 \leq t \leq 1}\left(g(t, s)+\sum_{i=1}^{m} \frac{\alpha_{i} t^{q-1}}{\Omega \Gamma\left(p_{i}+q\right)} g_{i}(\eta, s)\right) \\
& \leq g(s, s)+\sum_{i=1}^{m} \frac{\alpha_{i}}{\Omega \Gamma\left(p_{i}+q\right)} g_{i}(\eta, s) .
\end{aligned}
$$

To prove $\left(\mathrm{P}_{4}\right)$, by direct integration, we have

$$
\begin{aligned}
\int_{0}^{1} \max _{0 \leq t \leq 1} G(t, s) d s \leq & \int_{0}^{1}\left(g(s, s)+\sum_{i=1}^{m} \frac{\alpha_{i}}{\Omega \Gamma\left(p_{i}+q\right)} g_{i}(\eta, s)\right) d s \\
= & \int_{0}^{1} \frac{s^{q-1}(1-s)^{q-1}}{\Gamma(q)} d s \\
& +\sum_{i=1}^{m} \frac{\alpha_{i}}{\Omega \Gamma\left(p_{i}+q\right)}\left(\int_{\eta}^{1} \eta^{p_{i}+q-1}(1-s)^{q-1} d s\right. \\
& \left.+\int_{0}^{\eta}\left[\eta^{p_{i}+q-1}(1-s)^{q-1}-(\eta-s)^{p_{i}+q-1}\right] d s\right) \\
= & \frac{\Gamma(q)}{\Gamma(2 q)}+\sum_{i=1}^{m} \frac{\alpha_{i} \eta^{p_{i}+q-1}}{\Omega \Gamma\left(p_{i}+q\right)}\left(\frac{p_{i}+q(1-\eta)}{q\left(p_{i}+q\right)}\right) .
\end{aligned}
$$

To prove $\left(\mathrm{P}_{5}\right)$, from $g(t, s) \geq 0$ and $g_{i}(\eta, s) \geq 0, i=1,2, \ldots, m$, for all $0 \leq s, t \leq 1$, we have

$$
\begin{aligned}
\min _{\eta \leq t \leq 1} G(t, s) & =\min _{\eta \leq t \leq 1}\left(g(t, s)+\sum_{i=1}^{m} \frac{\alpha_{i} t^{q-1}}{\Omega \Gamma\left(p_{i}+q\right)} g_{i}(\eta, s)\right) \\
& \geq \min _{\eta \leq t \leq 1} g(t, s)+\min _{\eta \leq t \leq 1} \sum_{i=1}^{m} \frac{\alpha_{i} t^{q-1}}{\Omega \Gamma\left(p_{i}+q\right)} g_{i}(\eta, s) \\
& \geq \sum_{i=1}^{m} \frac{\alpha_{i} \eta^{q-1}}{\Omega \Gamma\left(p_{i}+q\right)} g_{i}(\eta, s)
\end{aligned}
$$

for $0 \leq s \leq 1$. This completes the proof.

Let $E=C([0,1], \mathbb{R})$ be the Banach space endowed with the supremum norm $\|\cdot\|$. Define the cone $\mathcal{P} \subset E$ by

$$
\mathcal{P}=\{u \in E: u(t) \geq 0\},
$$

and the operator $A: \mathcal{P} \rightarrow E$ by

$$
A u(t):=\int_{0}^{1} G(t, s) f(s, u(s)) d s .
$$


In view of Lemma 2.3, the positive solutions of problem (1.1)-(1.2) are given by the operator equation $u(t)=A u(t)$.

Lemma 2.5 Suppose that $f:[0,1] \times[0, \infty) \rightarrow[0, \infty)$ is continuous. The operator $A: \mathcal{P} \rightarrow$ $\mathcal{P}$ is completely continuous.

Proof Since $G(t, s) \geq 0$ for $s, t \in[0,1]$, we have $A u(t) \geq 0$ for all $u \in \mathcal{P}$. Hence, $A: \mathcal{P} \rightarrow \mathcal{P}$.

For a constant $R>0$, we define $\Phi=\{u \in \mathcal{P}:\|u\|<R\}$.

Let

$$
L=\max _{0 \leq t \leq 1,0 \leq u \leq R}|f(t, u)| .
$$

Then, for $u \in \Phi$, from Lemma 2.4, one has

$$
\begin{aligned}
|A u(t)| & =\left|\int_{0}^{1} G(t, s) f(s, u(s)) d s\right| \\
& \leq L \int_{0}^{1} G(t, s) d s \\
& \leq L \int_{0}^{1}\left(g(s, s)+\sum_{i=1}^{m} \frac{\alpha_{i}}{\Omega \Gamma\left(p_{i}+q\right)} g_{i}(\eta, s)\right) d s \\
& =L\left[\frac{\Gamma(q)}{\Gamma(2 q)}+\sum_{i=1}^{m} \frac{\alpha_{i} \eta^{p_{i}+q-1}}{\Omega \Gamma\left(p_{i}+q\right)}\left(\frac{p_{i}+q(1-\eta)}{q\left(p_{i}+q\right)}\right)\right]:=M .
\end{aligned}
$$

Therefore, $\|A u\| \leq M$, and so $A(\Phi)$ is uniformly bounded.

Now, we shall show that $A(\Phi)$ is equicontinuous. For $u \in \Phi, t_{1}, t_{2} \in[0,1], t_{1}<t_{2}$, we have

$$
\left|A u\left(t_{2}\right)-A u\left(t_{1}\right)\right| \leq L \int_{0}^{1}\left|G\left(t_{2}, s\right)-G\left(t_{1}, s\right)\right| d s,
$$

where $L$ is defined by $(2.10)$. Since $G(t, s)$ is continuous on $[0,1] \times[0,1]$, therefore $G(t, s)$ is uniformly continuous on $[0,1] \times[0,1]$. Hence, for any $\varepsilon>0$, there exists a positive constant

$$
\delta=\frac{1}{2}\left\{\frac{\varepsilon \Gamma(q)}{L}\left(\frac{1}{\frac{1}{q}+\sum_{i=1}^{m} \frac{\alpha_{i} \eta^{p_{i}+q-1}}{\Omega \Gamma\left(p_{i}+q\right)}\left(\frac{p_{i}+q(1-\eta)}{q\left(p_{i}+q\right)}\right)}\right)\right\}^{\frac{1}{q-1}}>0,
$$

whenever $\left|t_{2}-t_{1}\right|<\delta$, we have the following two cases.

Case $1 . \delta \leq t_{1}<t_{2}<1$.

Therefore,

$$
\begin{aligned}
\left|A u\left(t_{2}\right)-A u\left(t_{1}\right)\right| \leq & L \int_{0}^{1}\left|G\left(t_{2}, s\right)-G\left(t_{1}, s\right)\right| d s \\
= & L\left[\int_{0}^{t_{1}}\left|G\left(t_{2}, s\right)-G\left(t_{1}, s\right)\right| d s+\int_{t_{1}}^{t_{2}}\left|G\left(t_{2}, s\right)-G\left(t_{1}, s\right)\right| d s\right. \\
& \left.+\int_{t_{2}}^{1}\left|G\left(t_{2}, s\right)-G\left(t_{1}, s\right)\right| d s\right]
\end{aligned}
$$




$$
\begin{aligned}
& <\frac{\left(t_{2}^{q-1}-t_{1}^{q-1}\right) L}{\Gamma(q)}\left[\int_{0}^{1}(1-s)^{q-1} d s+\sum_{i=1}^{m} \frac{\alpha_{i}}{\Omega \Gamma\left(p_{i}+q\right)} \int_{0}^{1} g_{i}(\eta, s) d s\right] \\
& =\frac{\left(t_{2}^{q-1}-t_{1}^{q-1}\right) L}{\Gamma(q)}\left[\frac{1}{q}+\sum_{i=1}^{m} \frac{\alpha_{i} \eta^{p_{i}+q-1}}{\Omega \Gamma\left(p_{i}+q\right)}\left(\frac{p_{i}+q(1-\eta)}{q\left(p_{i}+q\right)}\right)\right] \\
& \leq \frac{(q-1) \delta^{q-1} L}{\Gamma(q)}\left[\frac{1}{q}+\sum_{i=1}^{m} \frac{\alpha_{i} \eta^{p_{i}+q-1}}{\Omega \Gamma\left(p_{i}+q\right)}\left(\frac{p_{i}+q(1-\eta)}{q\left(p_{i}+q\right)}\right)\right] \\
& <\varepsilon .
\end{aligned}
$$

Case 2. $0 \leq t_{1}<\delta, t_{2}<2 \delta$.

Therefore,

$$
\begin{aligned}
\left|A u\left(t_{2}\right)-A u\left(t_{1}\right)\right| & \leq L \int_{0}^{1}\left|G\left(t_{2}, s\right)-G\left(t_{1}, s\right)\right| d s \\
& <\frac{\left(t_{2}^{q-1}-t_{1}^{q-1}\right) L}{\Gamma(q)}\left[\frac{1}{q}+\sum_{i=1}^{m} \frac{\alpha_{i} \eta^{p_{i}+q-1}}{\Omega \Gamma\left(p_{i}+q\right)}\left(\frac{p_{i}+q(1-\eta)}{q\left(p_{i}+q\right)}\right)\right] \\
& \leq \frac{t_{2}^{q-1} L}{\Gamma(q)}\left[\frac{1}{q}+\sum_{i=1}^{m} \frac{\alpha_{i} \eta^{p_{i}+q-1}}{\Omega \Gamma\left(p_{i}+q\right)}\left(\frac{p_{i}+q(1-\eta)}{q\left(p_{i}+q\right)}\right)\right] \\
& <\frac{(2 \delta)^{q-1} L}{\Gamma(q)}\left[\frac{1}{q}+\sum_{i=1}^{m} \frac{\alpha_{i} \eta^{p_{i}+q-1}}{\Omega \Gamma\left(p_{i}+q\right)}\left(\frac{p_{i}+q(1-\eta)}{q\left(p_{i}+q\right)}\right)\right] \\
& =\varepsilon .
\end{aligned}
$$

Thus, $A(\Phi)$ is equicontinuous. In view of the Arzelá-Ascoli theorem, we have that $\overline{A(\Phi)}$ is compact, i.e., $A: \mathcal{P} \rightarrow \mathcal{P}$ is a completely continuous operator. This completes the proof.

For convenience, we set

$$
\begin{aligned}
& \Lambda_{1}=\sum_{i=1}^{m} \frac{\alpha_{i} \eta^{p_{i}+2(q-1)}}{\Omega \Gamma\left(p_{i}+q\right)}\left(\frac{p_{i}+q(1-\eta)}{q\left(p_{i}+q\right)}\right), \\
& \Lambda_{2}=\frac{\Gamma(q)}{\Gamma(2 q)}+\sum_{i=1}^{m} \frac{\alpha_{i} \eta^{p_{i}+q-1}}{\Omega \Gamma\left(p_{i}+q\right)}\left(\frac{p_{i}+q(1-\eta)}{q\left(p_{i}+q\right)}\right) \\
& \Lambda_{3}=\sum_{i=1}^{m} \frac{\alpha_{i} \eta^{p_{i}+2(q-1)}(1-\eta)^{q}}{\Omega \Gamma\left(p_{i}+q\right) q} .
\end{aligned}
$$

\section{Existence of at least one or two positive solutions}

For the main results of this section, we use the well-known Guo-Krasnoselskii fixed point theorem.

Theorem 3.1 ([20]) Let E be a Banach space, and let $\mathcal{P} \subset$ E be a cone. Assume that $\Omega_{1}, \Omega_{2}$ are open subsets of $E$ with $0 \in \Omega_{1}, \bar{\Omega}_{1} \subset \Omega_{2}$, and let $T: \mathcal{P} \cap\left(\bar{\Omega}_{2} \backslash \Omega_{1}\right) \rightarrow \mathcal{P}$ be a completely continuous operator such that:

(i) $\|T u\| \geq\|u\|, u \in \mathcal{P} \cap \partial \Omega_{1}$, and $\|T u\| \leq\|u\|, u \in \mathcal{P} \cap \partial \Omega_{2}$; or

(ii) $\|T u\| \leq\|u\|, u \in \mathcal{P} \cap \partial \Omega_{1}$, and $\|T u\| \geq\|u\|, u \in \mathcal{P} \cap \partial \Omega_{2}$.

Then $T$ has a fixed point in $\mathcal{P} \cap\left(\bar{\Omega}_{2} \backslash \Omega_{1}\right)$. 
Theorem 3.2 Let $f:[0,1] \times[0, \infty) \rightarrow[0, \infty)$ be a continuous function. Assume that there exist constants $r_{2}>r_{1}>0, \rho_{1} \in\left(\Lambda_{1}^{-1}, \infty\right)$ and $\rho_{2} \in\left(0, \Lambda_{2}^{-1}\right)$ such that:

$\left(\mathrm{H}_{1}\right) f(t, u) \geq \rho_{1} r_{1}$, for $(t, u) \in[0,1] \times\left[0, r_{1}\right]$;

$\left(\mathrm{H}_{2}\right) f(t, u) \leq \rho_{2} r_{2}$, for $(t, u) \in[0,1] \times\left[0, r_{2}\right]$.

Then boundary value problem (1.1)-(1.2) has at least one positive solution $u$ such that

$$
r_{1}<\|u\|<r_{2}
$$

Proof We shall show that the first part of Theorem 3.1 is satisfied. By Lemma 2.5, the operator $A: \mathcal{P} \rightarrow \mathcal{P}$ is completely continuous.

Let $\Phi_{1}=\left\{u \in E:\|u\|<r_{1}\right\}$, then for any $u \in \mathcal{P} \cap \partial \Phi_{1}$, we have $0 \leq u(t) \leq r_{1}$ for all $t \in$ $[0,1]$. From $\left(\mathrm{H}_{1}\right)$, it follows for $t \in[\eta, 1]$ that

$$
\begin{aligned}
(A u)(t)= & \int_{0}^{1} G(t, s) f(s, u(s)) d s \\
\geq & \int_{0}^{1} \min _{\eta \leq t \leq 1} G(t, s) f(s, u(s)) d s \\
\geq & \rho_{1} r_{1} \sum_{i=1}^{m} \frac{\alpha_{i} \eta^{q-1}}{\Omega \Gamma\left(p_{i}+q\right)} \int_{0}^{1} g_{i}(\eta, s) d s \\
= & \rho_{1} r_{1} \sum_{i=1}^{m} \frac{\alpha_{i} \eta^{q-1}}{\Omega \Gamma\left(p_{i}+q\right)}\left(\int_{\eta}^{1} \eta^{p_{i}+q-1}(1-s)^{q-1} d s\right. \\
& \left.+\int_{0}^{\eta}\left[\eta^{p_{i}+q-1}(1-s)^{q-1}-(\eta-s)^{p_{i}+q-1}\right] d s\right) \\
= & \rho_{1} r_{1}\left[\sum_{i=1}^{m} \frac{\alpha_{i} \eta^{p_{i}+2(q-1)}}{\Omega \Gamma\left(p_{i}+q\right)}\left(\frac{p_{i}+q(1-\eta)}{q\left(p_{i}+q\right)}\right)\right] \\
\geq & r_{1}=\|u\|,
\end{aligned}
$$

which yields

$$
\|A u\| \geq\|u\| \quad \text { for } u \in \mathcal{P} \cap \partial \Phi_{1} .
$$

Let $\Phi_{2}=\left\{u \in E:\|u\|<r_{2}\right\}$, then for any $u \in \mathcal{P} \cap \partial \Phi_{2}$, we have $0 \leq u(t) \leq r_{2}$ for all $t \in[0,1]$. For $t \in[0,1]$, assumption $\left(\mathrm{H}_{2}\right)$ yields

$$
\begin{aligned}
(A u)(t) & =\int_{0}^{1} G(t, s) f(s, u(s)) d s \\
& \leq \int_{0}^{1} G(s, s) f(s, u(s)) d s \\
& \leq \rho_{2} r_{2} \int_{0}^{1}\left(g(s, s)+\sum_{i=1}^{m} \frac{\alpha_{i}}{\Omega \Gamma\left(p_{i}+q\right)} g_{i}(\eta, s)\right) d s \\
& =\rho_{2} r_{2}\left[\frac{\Gamma(q)}{\Gamma(2 q)}+\sum_{i=1}^{m} \frac{\alpha_{i} \eta^{p_{i}+q-1}}{\Omega \Gamma\left(p_{i}+q\right)}\left(\frac{p_{i}+q(1-\eta)}{q\left(p_{i}+q\right)}\right)\right] \\
& \leq r_{2}=\|u\|,
\end{aligned}
$$


one has

$$
\|A u\| \leq\|u\| \quad \text { for } u \in \mathcal{P} \cap \partial \Phi_{2} .
$$

Therefore, from (3.1), (3.2) and the first part of Theorem 3.1, it follows that $A$ has a fixed point in $\mathcal{P} \cap\left(\Phi_{2} \backslash \Phi_{1}\right)$ which is a positive solution of boundary value problem (1.1)-(1.2). Hence, problem (1.1)-(1.2) has at least one positive solution $u$ such that

$$
r_{1}<\|u\|<r_{2}
$$

The proof is complete.

Theorem 3.3 Let all the assumptions of Theorem 3.2 hold. In addition, assume that

$\left(\mathrm{H}_{3}\right) \lim _{u \rightarrow \infty} \max _{t \in[0,1]} \frac{f(t, u)}{u}=\infty$.

Then boundary value problem (1.1)-(1.2) has at least two positive solutions $u_{1}$ and $u_{2}$ such that

$$
0<r_{1}<\left\|u_{1}\right\|<r_{2}<\left\|u_{2}\right\|
$$

Proof It follows from Theorem 3.2 that there exists a positive solution $u_{1}$ such that $r_{1}<$ $\left\|u_{1}\right\|<r_{2}$. From $\left(\mathrm{H}_{3}\right)$, there exists $r^{*}>r_{2}$ such that for any $t \in[0,1]$ and for any $M^{*} \in$ $\left(\Lambda_{1}^{-1}, \infty\right)$,

$$
f(t, u) \geq M^{*} u \quad \text { for } u \geq r^{*}
$$

Let $\Phi_{3}=\left\{u \in E:\|u\|<r^{*}\right\}$. Then, for any $u \in \mathcal{P} \cap \partial \Phi_{3}$ and for $t \in[\eta, 1]$, we have

$$
\begin{aligned}
(A u)(t) & =\int_{0}^{1} G(t, s) f(s, u(s)) d s \\
& \geq \int_{0}^{1} \min _{\eta \leq t \leq 1} G(t, s) f(s, u(s)) d s \\
& \geq M^{*} r^{*} \sum_{i=1}^{m} \frac{\alpha_{i} \eta^{q-1}}{\Omega \Gamma\left(p_{i}+q\right)} \int_{0}^{1} g_{i}(\eta, s) d s \\
& =M^{*} r^{*}\left[\sum_{i=1}^{m} \frac{\alpha_{i} \eta^{p_{i}+2(q-1)}}{\Omega \Gamma\left(p_{i}+q\right)}\left(\frac{p_{i}+q(1-\eta)}{q\left(p_{i}+q\right)}\right)\right] \\
& \geq r^{*}=\|u\| .
\end{aligned}
$$

This implies that

$$
\|A u\| \geq\|u\| \quad \text { for } u \in \mathcal{P} \cap \partial \Phi_{3} .
$$

It follows from (3.2), (3.3) and the second part of Theorem 3.1 that $A$ has a fixed point in $\mathcal{P} \cap\left(\bar{\Phi}_{3} \backslash \Phi_{2}\right)$. 
Therefore, we conclude that boundary value problem (1.1)-(1.2) has at least two positive solutions such that

$$
0<r_{1}<\left\|u_{1}\right\|<r_{2}<\left\|u_{2}\right\|
$$

Similarly to the previous theorems, we can prove the following.

Theorem 3.4 Let $f:[0,1] \times[0, \infty) \rightarrow[0, \infty)$ be a continuous function. Assume that there exist constants $0<r_{1}<r_{2}$ and $\rho_{1} \in\left(\Lambda_{1}^{-1}, \infty\right), \rho_{2} \in\left(0, \Lambda_{2}^{-1}\right)$ such that:

$\left(\mathrm{H}_{4}\right) f(t, u) \leq \rho_{2} r_{1}$ for $(t, u) \in[0,1] \times\left[0, r_{1}\right]$;

$\left(\mathrm{H}_{5}\right) f(t, u) \geq \rho_{1} r_{2}$ for $(t, u) \in[0,1] \times\left[0, r_{2}\right]$;

$\left(\mathrm{H}_{6}\right) \lim _{u \rightarrow \infty} \max _{t \in[0,1]} \frac{f(t, u)}{u}=0$.

Then boundary value problem (1.1)-(1.2) has at least two positive solutions $u_{1}$ and $u_{2}$ such that

$$
0<r_{1}<\left\|u_{1}\right\|<r_{2}<\left\|u_{2}\right\|
$$

Corollary 3.1 Assume that conditions $\left(\mathrm{H}_{4}\right)-\left(\mathrm{H}_{5}\right)$ are satisfied. Then boundary value problem (1.1)-(1.2) has at least one positive solution $u$ such that

$$
r_{1}<\|u\|<r_{2}
$$

\section{Existence of at least three positive solutions}

In this section we use the Leggett-Williams fixed point theorem to prove the existence of at least three positive solutions.

Definition 4.1 A continuous mapping $\theta: \mathcal{P} \rightarrow[0,+\infty)$ is said to be a nonnegative continuous concave functional on the cone $\mathcal{P}$ of a real Banach space $E$ provided that

$$
\theta(\lambda u+(1-\lambda) v) \geq \lambda \theta(u)+(1-\lambda) \theta(v)
$$

for all $u, v \in \mathcal{P}$ and $\lambda \in[0,1]$.

Let $a, b, d>0$ be constants. We define $\mathcal{P}_{d}=\{u \in \mathcal{P}:\|u\|<d\}, \overline{\mathcal{P}}_{d}=\{u \in \mathcal{P}:\|u\| \leq d\}$ and $\mathcal{P}(\theta, a, b)=\{u \in \mathcal{P}: \theta(u) \geq a,\|u\| \leq b\}$.

Theorem 4.1 ([21]) Let $\mathcal{P}$ be a cone in the real Banach space $E$ and $c>0$ be a constant. Assume that there exists a concave nonnegative continuous functional $\theta$ on $\mathcal{P}$ with $\theta(u) \leq$ $\|u\|$ for all $u \in \overline{\mathcal{P}}_{c}$. Let $A: \overline{\mathcal{P}}_{c} \rightarrow \overline{\mathcal{P}}_{c}$ be a completely continuous operator. Suppose that there exist constants $0<a<b<d \leq c$ such that the following conditions hold:

(i) $\{u \in \mathcal{P}(\theta, b, d): \theta(u)>b\} \neq \emptyset$ and $\theta(A u)>b$ for $u \in \mathcal{P}(\theta, b, d)$;

(ii) $\|A u\|<a$ for $u \leq a$;

(iii) $\theta(A u)>b$ for $u \in \mathcal{P}(\theta, b, c)$ with $\|A u\|>d$.

Then $A$ has at least three fixed points $u_{1}, u_{2}$ and $u_{3}$ in $\overline{\mathcal{P}}_{c}$.

Furthermore, $\left\|u_{1}\right\|<a, b<\theta\left(u_{2}\right), a<\left\|u_{3}\right\|$ with $\theta\left(u_{3}\right)<b$. 
We now prove the following result.

Theorem 4.2 Let $f:[0,1] \times[0, \infty) \rightarrow[0, \infty)$ be a continuous function. Suppose that there exist constants $0<a<b<c$ such that the following assumptions hold:

$\left(\mathrm{H}_{7}\right) f(t, u)<\Lambda_{2}^{-1} a$ for $(t, u) \in[0,1] \times[0, a]$;

$\left(\mathrm{H}_{8}\right) f(t, u)>\Lambda_{3}^{-1} b$ for $(t, u) \in[\eta, 1] \times[b, c]$;

$\left(\mathrm{H}_{9}\right) f(t, u) \leq \Lambda_{2}^{-1} c$ for $(t, u) \in[0,1] \times[0, c]$.

Then boundary value problem (1.1)-(1.2) has at least three positive solutions $u_{1}, u_{2}$ and $u_{3}$ with

$$
\left\|u_{1}\right\|<a, \quad b<\min _{\eta \leq t \leq 1} u_{2}(t)
$$

and

$$
a<\left\|u_{3}\right\| \quad \text { with } \min _{\eta \leq t \leq 1} u_{3}(t)<b .
$$

Proof We will show that all the conditions of the Leggett-Williams fixed point theorem are satisfied for the operator $A$ defined by (2.9).

For $u \in \overline{\mathcal{P}}_{c}$, we have $\|u\| \leq c$. From condition $\left(\mathrm{H}_{9}\right)$, we have $f(t, u(t)) \leq \Lambda_{2}^{-1} c$ for $t \in[0,1]$. Therefore,

$$
\begin{aligned}
(A u)(t) & =\int_{0}^{1} G(t, s) f(s, u(s)) d s \\
& \leq \int_{0}^{1} G(s, s) f(s, u(s)) d s \\
& \leq \Lambda_{2}^{-1} c \int_{0}^{1}\left(g(s, s)+\sum_{i=1}^{m} \frac{\alpha_{i}}{\Omega \Gamma\left(p_{i}+q\right)} g_{i}(\eta, s)\right) d s \\
& =\Lambda_{2}^{-1} c\left[\frac{\Gamma(q)}{\Gamma(2 q)}+\sum_{i=1}^{m} \frac{\alpha_{i} \eta^{p_{i}+q-1}}{\Omega \Gamma\left(p_{i}+q\right)}\left(\frac{p_{i}+q(1-\eta)}{q\left(p_{i}+q\right)}\right)\right] \\
& =c,
\end{aligned}
$$

which implies $\|A u\| \leq c$. Hence, $A: \overline{\mathcal{P}}_{c} \rightarrow \overline{\mathcal{P}}_{c}$.

If $u \in \overline{\mathcal{P}}_{a}$, then condition $\left(\mathrm{H}_{7}\right)$ yields

$$
\begin{aligned}
(A u)(t) & <\Lambda_{2}^{-1} a \int_{0}^{1}\left(g(s, s)+\sum_{i=1}^{m} \frac{\alpha_{i}}{\Omega \Gamma\left(p_{i}+q\right)} g_{i}(\eta, s)\right) d s \\
& =\Lambda_{2}^{-1} a\left[\frac{\Gamma(q)}{\Gamma(2 q)}+\sum_{i=1}^{m} \frac{\alpha_{i} \eta^{p_{i}+q-1}}{\Omega \Gamma\left(p_{i}+q\right)}\left(\frac{p_{i}+q(1-\eta)}{q\left(p_{i}+q\right)}\right)\right] \\
& =a .
\end{aligned}
$$

Thus $\|A u\|<a$. Therefore, condition (ii) of Theorem 4.1 holds.

Define a concave nonnegative continuous functional $\theta$ on $\mathcal{P}$ by $\theta(u)=\min _{t \in[\eta, 1]}|u(t)|$. To check condition (i) of Theorem 4.1, we choose $u(t)=(b+c) / 2$ for $t \in[0,1]$. It is easy to see that $u(t)=(b+c) / 2 \in \mathcal{P}(\theta, b, c)$ and $\theta(u)=\theta((b+c) / 2)>b$; consequently, the set 
$\{u \in \mathcal{P}(\theta, b, c): \theta(u)>b\} \neq \emptyset$. Hence, if $u \in \mathcal{P}(\theta, b, c)$, then $b \leq u(t) \leq c$ for $t \in[\eta, 1]$. From condition $\left(\mathrm{H}_{8}\right)$, we have

$$
\begin{aligned}
\theta(A u) & =\min _{\eta \leq t \leq 1}|(A u)(t)| \\
& \geq \int_{\eta}^{1} \min _{\eta \leq t \leq 1} G(t, s) f(s, u(s)) d s \\
& >\Lambda_{3}^{-1} b \sum_{i=1}^{m} \frac{\alpha_{i} \eta^{q-1}}{\Omega \Gamma\left(p_{i}+q\right)} \int_{\eta}^{1} g_{i}(\eta, s) d s \\
& =\Lambda_{3}^{-1} b\left[\sum_{i=1}^{m} \frac{\alpha_{i} \eta^{p_{i}+2(q-1)}(1-\eta)^{q}}{\Omega \Gamma\left(p_{i}+q\right) q}\right] \\
& =b .
\end{aligned}
$$

Thus $\theta(A u)>b$ for all $u \in \mathcal{P}(\theta, b, c)$. This shows that condition (i) of Theorem 4.1 is also satisfied.

We finally show that condition (iii) of Theorem 4.1 also holds. Assume that $u \in \mathcal{P}(\theta, b, c)$ with $\|A u\|>d$, then we have $b \leq u(t) \leq c$ for all $t \in[\eta, 1]$. From $\left(\mathrm{H}_{8}\right)$ and Lemma 2.4, one has

$$
\begin{aligned}
\theta(A u) & =\min _{\eta \leq t \leq 1}|(A u)(t)| \\
& >\Lambda_{3}^{-1} b \sum_{i=1}^{m} \frac{\alpha_{i} \eta^{q-1}}{\Omega \Gamma\left(p_{i}+q\right)} \int_{\eta}^{1} g_{i}(\eta, s) d s \\
& =\Lambda_{3}^{-1} b\left[\sum_{i=1}^{m} \frac{\alpha_{i} \eta^{p_{i}+2(q-1)}(1-\eta)^{q}}{\Omega \Gamma\left(p_{i}+q\right) q}\right] \\
& =b .
\end{aligned}
$$

So, condition (iii) of Theorem 4.1 is satisfied. Therefore, an application of Theorem 4.1 implies that boundary value problem (1.1)-(1.2) has at least three positive solutions $u_{1}, u_{2}$ and $u_{3}$ such that

$$
\left\|u_{1}\right\|<a, \quad b<\min _{\eta \leq t \leq 1}\left|u_{2}(t)\right| \text { and } \quad a<\left\|u_{3}\right\| \quad \text { with } \min _{\eta \leq t \leq 1}\left|u_{3}(t)\right|<b \text {. }
$$

The proof is complete.

\section{Examples}

In this section, we present some examples to illustrate our results.

Example 5.1 Consider the following three-point three-term fractional integral boundary value problem:

$$
\begin{aligned}
& D^{\frac{3}{2}} u(t)+f(t, u)=0, \quad t \in(0,1), \\
& u(0)=0, \quad u(1)=\sqrt{3}\left(I^{\frac{\pi}{2}} u\right)\left(\frac{1}{4}\right)+\frac{\pi}{2}\left(I^{\frac{3}{2}} u\right)\left(\frac{1}{4}\right)+2\left(I^{\frac{2}{3}} u\right)\left(\frac{1}{4}\right),
\end{aligned}
$$


where

$$
f(t, u)= \begin{cases}u(1-u)+6(1+t) ; & 0 \leq t \leq 1 ; 0 \leq u \leq 1 \\ 6(1+t) e^{1-u}+\sin ^{2}(u \pi) ; & 0 \leq t \leq 1 ; 1 \leq u<\infty\end{cases}
$$

Set $m=3, \eta=1 / 4, q=3 / 2, p_{1}=\pi / 2, p_{2}=3 / 2, p_{3}=2 / 3, \alpha_{1}=\sqrt{3}, \alpha_{2}=\pi / 2, \alpha_{3}=2$, and we can show that

$$
\Omega=1-\sum_{i=1}^{m} \frac{\alpha_{i} \eta^{p_{i}+q-1} \Gamma(q)}{\Gamma\left(p_{i}+q\right)} \approx 0.590859 .
$$

Through a simple calculation we can get

$$
\begin{aligned}
& \Lambda_{1}=\sum_{i=1}^{m} \frac{\alpha_{i} \eta^{p_{i}+2(q-1)}}{\Omega \Gamma\left(p_{i}+q\right)}\left(\frac{p_{i}+q(1-\eta)}{q\left(p_{i}+q\right)}\right) \approx 0.218023 \\
& \Lambda_{2}=\frac{\Gamma(q)}{\Gamma(2 q)}+\sum_{i=1}^{m} \frac{\alpha_{i} \eta^{p_{i}+q-1}}{\Omega \Gamma\left(p_{i}+q\right)}\left(\frac{p_{i}+q(1-\eta)}{q\left(p_{i}+q\right)}\right) \approx 0.879159 .
\end{aligned}
$$

Choose $r_{1}=1, r_{2}=14, \rho_{1}=5$ and $\rho_{2}=1$, then $f(t, u)$ satisfies

$$
f(t, u) \geq 6 \geq 5=\rho_{1} r_{1}, \quad \forall(t, u) \in[0,1] \times[0,1]
$$

and

$$
f(t, u) \leq 13 \leq 14=\rho_{2} r_{2}, \quad \forall(t, u) \in[0,1] \times[0,14] .
$$

Thus, $\left(\mathrm{H}_{1}\right)$ and $\left(\mathrm{H}_{2}\right)$ hold. By Theorem 3.2, we have that boundary value problem (5.1)(5.2) has at least one positive solution $u$ such that $1<\|u\|<14$.

Example 5.2 Consider the following three-point four-term fractional integral boundary value problem:

$$
\begin{aligned}
& D^{\frac{3}{2}} u(t)+f(t, u)=0, \quad t \in(0,1), \\
& \left\{\begin{array}{l}
u(0)=0, \\
u(1)=\frac{1}{6}\left(I^{\frac{1}{2}} u\right)\left(\frac{2}{3}\right)+\frac{\sqrt{3}}{2}\left(I^{\sqrt{2}} u\right)\left(\frac{2}{3}\right)+\frac{1}{2}\left(I^{\sqrt{\pi}} u\right)\left(\frac{2}{3}\right)+\frac{\sqrt{\pi}}{6}\left(I^{\frac{5}{6}} u\right)\left(\frac{2}{3}\right),
\end{array}\right.
\end{aligned}
$$

where

$$
f(t, u)= \begin{cases}u^{2}\left(\frac{1}{2}-u\right)+2\left(1+t^{2}\right) ; & 0 \leq t \leq 1 ; 0 \leq u \leq 1 / 2 \\ 4\left(1+t^{2}\right) \cos ^{2}\left(\frac{u \pi}{2}\right)+\frac{1}{95}\left(u-\frac{1}{2}\right)^{2} ; & 0 \leq t \leq 1 ; 1 / 2 \leq u<\infty\end{cases}
$$

Here $m=4, \eta=2 / 3, q=3 / 2, p_{1}=1 / 2, p_{2}=\sqrt{2}, p_{3}=\sqrt{\pi}, p_{4}=5 / 6, \alpha_{1}=1 / 6, \alpha_{2}=\sqrt{3} / 2$, $\alpha_{3}=1 / 2, \alpha_{4}=\sqrt{\pi} / 6$, and we can show that

$$
\Omega=1-\sum_{i=1}^{m} \frac{\alpha_{i} \eta^{p_{i}+q-1} \Gamma(q)}{\Gamma\left(p_{i}+q\right)} \approx 0.515010
$$


Through a simple calculation we can get

$$
\begin{aligned}
& \Lambda_{1}=\sum_{i=1}^{m} \frac{\alpha_{i} \eta^{p_{i}+2(q-1)}}{\Omega \Gamma\left(p_{i}+q\right)}\left(\frac{p_{i}+q(1-\eta)}{q\left(p_{i}+q\right)}\right) \approx 0.351492, \\
& \Lambda_{2}=\frac{\Gamma(q)}{\Gamma(2 q)}+\sum_{i=1}^{m} \frac{\alpha_{i} \eta^{p_{i}+q-1}}{\Omega \Gamma\left(p_{i}+q\right)}\left(\frac{p_{i}+q(1-\eta)}{q\left(p_{i}+q\right)}\right) \approx 0.873602 .
\end{aligned}
$$

Choose $r_{1}=1 / 2, r_{2}=10, \rho_{1}=3$ and $\rho_{2}=1$, then $f(t, u)$ satisfies

$$
f(t, u) \geq 2 \geq \frac{3}{2}=\rho_{1} r_{1}, \quad \forall(t, u) \in[0,1] \times[0,1 / 2]
$$

and

$$
f(t, u) \leq 9 \leq 10=\rho_{2} r_{2}, \quad \forall(t, u) \in[0,1] \times[0,10],
$$

and

$$
\lim _{u \rightarrow \infty} \max _{t \in[0,1]} \frac{f(t, u)}{u}=\lim _{u \rightarrow \infty} \max _{t \in[0,1]}\left[\frac{4\left(1+t^{2}\right) \cos ^{2}(u \pi / 2)+(1 / 95)(u-1 / 2)^{2}}{u}\right]=\infty
$$

Thus, $\left(\mathrm{H}_{1}\right),\left(\mathrm{H}_{2}\right)$ and $\left(\mathrm{H}_{3}\right)$ hold. By Theorem 3.3, we have that boundary value problem (5.3)-(5.4) has at least two positive solutions $u_{1}$ and $u_{2}$ such that $0<1 / 2<\left\|u_{1}\right\|<10<\left\|u_{2}\right\|$.

Example 5.3 Consider the following three-point five-term fractional integral boundary value problem:

$$
\begin{aligned}
& D^{\frac{3}{2}} u(t)+f(t, u)=0, \quad t \in(0,1), \\
& \left\{\begin{aligned}
u(0)= & 0, \\
u(1)= & \frac{1}{2}\left(I^{\frac{1}{2}} u\right)\left(\frac{1}{2}\right)+\frac{4}{3}\left(I^{\frac{3}{2}} u\right)\left(\frac{1}{2}\right)+\frac{\sqrt{\pi}}{4}\left(I^{\frac{5}{2}} u\right)\left(\frac{1}{2}\right) \\
& +\frac{5}{6}\left(I^{\frac{7}{2}} u\right)\left(\frac{1}{2}\right)+\frac{\sqrt{3}}{2}\left(I^{\frac{9}{2}} u\right)\left(\frac{1}{2}\right),
\end{aligned}\right.
\end{aligned}
$$

where

$$
f(t, u)= \begin{cases}u\left(\frac{3}{4}-u\right)+\frac{1}{4}(t+1) ; & 0 \leq t \leq 1 ; 0 \leq u \leq 3 / 4 \\ \frac{1}{2}(t+1) \cos ^{2}(u \pi)+43\left(u-\frac{3}{4}\right)^{2} ; & 0 \leq t \leq 1 ; 3 / 4 \leq u \leq 5 / 4 \\ \frac{1}{4}(t+44)+\sin ^{2}\left(u-\frac{5}{4}\right) \pi ; & 0 \leq t \leq 1 ; 5 / 4 \leq u<\infty\end{cases}
$$

Set $m=5, \eta=1 / 2, q=3 / 2, p_{1}=1 / 2, p_{2}=3 / 2, p_{3}=5 / 2, p_{4}=7 / 2, p_{5}=9 / 2, \alpha_{1}=1 / 2$, $\alpha_{2}=4 / 3, \alpha_{3}=\sqrt{\pi} / 4, \alpha_{4}=5 / 6, \alpha_{5}=\sqrt{3} / 2$, and we can show that

$$
\Omega=1-\sum_{i=1}^{m} \frac{\alpha_{i} \eta^{p_{i}+q-1} \Gamma(q)}{\Gamma\left(p_{i}+q\right)} \approx 0.620434
$$

Through a simple calculation we can get

$$
\Lambda_{2}=\frac{\Gamma(q)}{\Gamma(2 q)}+\sum_{i=1}^{m} \frac{\alpha_{i} \eta^{p_{i}+q-1}}{\Omega \Gamma\left(p_{i}+q\right)}\left(\frac{p_{i}+q(1-\eta)}{q\left(p_{i}+q\right)}\right) \approx 0.755575
$$




$$
\Lambda_{3}=\sum_{i=1}^{m} \frac{\alpha_{i} \eta^{p_{i}+2(q-1)}(1-\eta)^{q}}{\Omega \Gamma\left(p_{i}+q\right) q} \approx 0.115052 .
$$

Choose $a=3 / 4, b=5 / 4$ and $c=10$, then $f(t, u)$ satisfies

$$
f(t, u) \leq \frac{41}{64}<0.992622 \approx \Lambda_{2}^{-1} a, \quad \forall(t, u) \in[0,1] \times[0,3 / 4]
$$

and

$$
f(t, u) \geq \frac{89}{8}>10.864653 \approx \Lambda_{3}^{-1} b, \quad \forall(t, u) \in[1 / 2,1] \times[5 / 4,10],
$$

and

$$
f(t, u) \leq \frac{49}{4} \leq 13.234954=\Lambda_{2}^{-1} c, \quad \forall(t, u) \in[0,1] \times[0,10] .
$$

Thus, $\left(\mathrm{H}_{7}\right)$, $\left(\mathrm{H}_{8}\right)$ and $\left(\mathrm{H}_{9}\right)$ hold. By Theorem 4.2 , we have that boundary value problem (5.5)-(5.6) has at least three positive solutions $u_{1}, u_{2}$ and $u_{3}$ such that $\left\|u_{1}\right\|<3 / 4,5 / 4<$ $\min _{(1 / 2) \leq t \leq 1} u_{2}(t)$ and $3 / 4<\left\|u_{3}\right\|$ with $\min _{(1 / 2) \leq t \leq 1} u_{3}(t)<5 / 4$.

\section{Competing interests}

The authors declare that they have no competing interests.

\section{Authors' contributions}

All authors contributed equally in this article. They read and approved the final manuscript.

\section{Author details}

'Department of Mathematics, Faculty of Applied Science, King Mongkut's University of Technology North Bangkok, Bangkok, Thailand. ${ }^{2}$ Department of Mathematics, University of loannina, loannina, 451 10, Greece.

\section{Authors' information}

Sotiris K Ntouyas is a member of Nonlinear Analysis and Applied Mathematics (NAAM) - Research Group at King Abdulaziz University, Jeddah, Saudi Arabia.

\section{Acknowledgements}

We would like to thank the reviewers for their valuable comments and suggestions on the manuscript. This research of J Tariboon and W Sudsutad is supported by King Mongkut's University of Technology North Bangkok, Thailand.

\section{Received: 19 August 2013 Accepted: 6 January 2014 Published: 22 Jan 2014}

\section{References}

1. Miller, KS, Ross, B: An Introduction to the Fractional Calculus and Differential Equations. Wiley, New York (1993)

2. Samko, SG, Kilbas, AA, Marichev, Ol: Fractional Integrals and Derivatives: Theory and Applications. Gordon \& Breach, Yverdon (1993)

3. Podlubny, I: Fractional Differential Equations. Academic Press, San Diego (1999)

4. Kilbas, AA, Srivastava, HM, Trujillo, Jj: Theory and Applications of Fractional Differential Equations. North-Holland Mathematics Studies, vol. 204. Elsevier, Amsterdam (2006)

5. Lakshmikantham, V, Leela, S, Vasundhara Devi, J: Theory of Fractional Dynamic Systems. Cambridge Academic Publishers, Cambridge (2009)

6. Baleanu, D, Diethelm, K, Scalas, E, Trujillo, J: Fractional Calculus Models and Numerical Methods. Series on Complexity, Nonlinearity and Chaos. World Scientific, Boston (2012)

7. Diethelm, K: The Analysis of Fractional Differential Equations. An Application-Oriented Exposition Using Differential Operators of Caputo Type. Lecture Notes in Mathematics, vol. 2004. Springer, Berlin (2010)

8. Guezane-Lakoud, A, Khaldi, R: Solvability of a three-point fractional nonlinear boundary value problem. Differ. Equ. Dyn. Syst. 20, 395-403 (2012)

9. Guezane-Lakoud, A, Khaldi, R: Positive solution to a higher order fractional boundary value problem with fractional integral condition. Rom. J. Math. Comput. Sci. 2, 41-54 (2012)

10. Kaufmann, E: Existence and nonexistence of positive solutions for a nonlinear fractional boundary value problem. Discrete Contin. Dyn. Syst. 2009, suppl., 416-423 (2009)

11. Wang, J, Xiang, H, Liu, Z: Positive solution to nonzero boundary values problem for a coupled system of nonlinear fractional differential equations. Int. J. Differ. Equ. 2010, Article ID 186928 (2010)

12. Bai, Z, Lü, H: Positive solutions for boundary value problem of nonlinear fractional differential equation. J. Math. Anal. Appl. 311, 495-505 (2005) 
13. Bai, Z: On positive solutions of a nonlocal fractional boundary value problem. Nonlinear Anal. 72, 916-924 (2010)

14. Sudsutad, W, Tariboon, J: Boundary value problems for fractional differential equations with three-point fractional integral boundary conditions. Adv. Differ. Equ. 2012, 93 (2012)

15. Ntouyas, SK: Existence results for nonlocal boundary value problems for fractional differential equations and inclusions with fractional integral boundary conditions. Discuss. Math., Differ. Incl. Control Optim. 33, 17-39 (2013)

16. Ntouyas, SK: Boundary value problems for nonlinear fractional differential equations and inclusions with nonlocal and fractional integral boundary conditions. Opusc. Math. 33, 117-138 (2013)

17. Guezane-Lakoud, A, Khaldi, R: Solvability of a fractional boundary value problem with fractional integral condition. Nonlinear Anal. 75, 2692-2700 (2012)

18. Yang, W: Positive solutions for nonlinear Caputo fractional differential equations with integral boundary conditions J. Appl. Math. Comput. (2014). doi:10.1007/s12190-013-0679-8

19. Ahmad, B, Ntouyas, SK, Assolani, A: Caputo type fractional differential equations with nonlocal Riemann-Liouville integral boundary conditions. J. Appl. Math. Comput. 41, 339-350 (2013)

20. Guo, D, Lakshmikantham, V: Nonlinear Problems in Abstract Cones. Academic Press, New York (1988)

21. Leggett, RW, Williams, LR: Multiple positive fixed points of nonlinear operators on ordered Banach spaces. Indiana Univ. Math. J. 28, 673-688 (1979)

10.1186/1687-1847-2014-28

Cite this article as: Tariboon et al.: Positive solutions for fractional differential equations with three-point multi-term fractional integral boundary conditions. Advances in Difference Equations 2014, 2014:28

\section{Submit your manuscript to a SpringerOpen ${ }^{\circ}$ journal and benefit from:}

- Convenient online submission

Rigorous peer review

- Immediate publication on acceptance

- Open access: articles freely available online

- High visibility within the field

- Retaining the copyright to your article 